\title{
Prognostic implication of molecular aberrations in cytogenetically normal acute myeloid leukemia patients receiving allogeneic hematopoietic stem cell transplantation
}

\author{
Y.C. Liu ${ }^{1,2,3 *}$, H.H. Hsia $0^{1,2 *}$, P.M. Lin ${ }^{4}$, W.C. Yang ${ }^{1,3}$, C.S. Chang ${ }^{1,2}$, \\ T.C. Liu ${ }^{1,3}$, J.F. Hsu', M.Y. Yang ${ }^{5}$ and S.F. Lin ${ }^{1,2}$ \\ ${ }^{1}$ Division of Hematology-Oncology, Department of Internal Medicine, \\ Kaohsiung Medical University Hospital, Kaohsiung, Taiwan \\ ${ }^{2}$ Department of Internal Medicine, Faculty of Medicine, College of Medicine, \\ Kaohsiung Medical University, Kaohsiung, Taiwan \\ ${ }^{3}$ Graduate Institute of Medicine, College of Medicine, \\ Kaohsiung Medical University, Kaohsiung, Taiwan \\ ${ }^{4}$ Department of Nursing, I-Shou University, Kaohsiung, Taiwan \\ ${ }^{5}$ Graduate Institute of Clinical Medical Sciences, College of Medicine, \\ Chang Gung University, Tao-Yuan, Taiwan \\ *These authors contributed equally to this study. \\ Corresponding author: S.F. Lin \\ E-mail: shlin@kmu.edu.tw
}

Genet. Mol. Res. 12 (4): 5414-5423 (2013)

Received February 26, 2013

Accepted September 2, 2013

Published November 11, 2013

DOI http://dx.doi.org/10.4238/2013.November.11.3

\begin{abstract}
Different molecular aberrations can be discriminated into certain prognostic subgroups in cytogenetically normal acute myeloid leukemia (CN-AML) patients but their impact on allogeneic hematopoietic stem cell transplantation (allo-HSCT) remains controversial and studies from Asian populations are lacking. Fortytwo adult non-M3 AML patients receiving allo-HSCT from 2002 to 2009 in southern Taiwan were retrospectively reviewed for survey,
\end{abstract}


23 (54.7\%) of whom were CN-AML. NPM1, FLT3-ITD, and CEBPA were analyzed. After a median follow-up of 104 weeks (range, 8 to 384), patients in the good risk group (harboring either $N P M 1$ or CEBPA mutation without concurrent FLT3-ITD) showed a borderline worse overall survival (OS) compared with the intermediate/poor risk group $(\mathrm{P}=0.08)$. Interestingly, a poorer OS was found in patients with the CEBPA mutation $(\mathrm{P}=0.003)$ but not the NPM1 mutation $(\mathrm{P}=0.96)$. No OS difference was found between patients with or without FLT3-ITD $(\mathrm{P}=0.15)$. In patients receiving allo-HSCT at first remission, there was no significant OS benefit in the good risk group $(\mathrm{P}=0.33)$. In patients receiving allo-HSCT beyond first remission, disease status played a major role $(\mathrm{P}=0.006)$, irrespective of molecular aberrations. Allo-HSCT in good risk patients should be carefully evaluated in Taiwanese, especially in patients with the CEBPA mutation. Conversely, allo-HSCT should be considered in first remission in patients with an intermediate/poor risk, where it may overcome the adverse impact of FLT3-ITD. Disease status remained a main issue in patients receiving allo-HSCT beyond first remission.

Key words: Acute myeloid leukemia; Cytogenetic; Mutation; Hematopoietic stem cell transplantation; Molecular aberrations

\section{INTRODUCTION}

Cytogenetics is one of the most important prognostic factors in acute myeloid leukemia (AML), and the categorization into good, intermediate, and poor risk groups has been widely used for risk-adapted therapy (Grimwade et al., 1998; Byrd et al., 2002; Mrozek et al., 2004). Cytogenetically normal AML (CN-AML), found in 40-50\% of all cases of AML, represents a heterogenous entity and is categorized as an intermediate risk group. In recent years, several molecular aberrations have been found to refine the prognosis and have changed the understanding of CN-AML. Among them, the mutations of FLT3 (Fmslike tyrosine kinase 3) (Fröhling et al., 2002; Thiede et al., 2002), NPM1 (nucleophosmin 1) (Döhner et al., 2005; Schnittger et al., 2005), and CEBPA (CCAAT enhancer-binding protein $\alpha$ ) (Fröhling et al., 2004; Green et al., 2010; Taskesen et al., 2011), have been found to have a prognostic impact and have been integrated into risk stratification, for example, in the National Comprehensive Cancer Network (NCCN) guidelines and European LeukemiaNet (ELN) recommendations (Döhner et al., 2010; O'Donnell et al., 2011). In CN-AML patients, isolated NPM1 or CEBPA mutations without FLT3-ITD are considered to be a good risk and FLT3-ITD a poor risk in the NCCN guidelines but an intermediate risk in the ELN recommendations (Döhner et al., 2010; Foran, 2010; O’Donnell et al., 2011). The combination of morphology, immunophenotyping, cytogenetics, and molecular aberrations has provided essential parameters for treatment decision making, especially in post-remission therapy, including consolidation chemotherapy and allogeneic hematopoietic stem cell transplantation (allo-HSCT).

Allo-HSCT from a suitable donor after myeloablative conditioning is a curative 
treatment option for younger AML patients in first remission. Based on cytogenetic risk stratification, allo-HSCT has shown significant relapse-free survival and overall survival benefits for patients with intermediate and poor risk AML but not for those with good risk AML, according to a meta-analysis of prospective trials compared with non-transplant postremission therapies (Koreth et al., 2009). However, the impact of molecular aberrations on allo-HSCT remains unclear. In patients with FLT3-ITD, no survival benefits from alloHSCT were found based on a "donor versus no donor" comparison (Gale et al., 2005), but a reduced risk of relapse was found in patients with FLT3-ITD after allo-HSCT (Bornhäuser et al., 2007). In patients with isolated NPM1 mutations without FLT3-ITD, no survival benefits were found based on a "donor versus no-donor" comparison (Schlenk et al., 2008). In patients with isolated CEBPA mutations, the survival benefit from allo-HSCT is currently unknown. Furthermore, most studies have been in Western populations, and studies from Asian population are still lacking.

In patients with relapsed disease, the prognosis remains poor and long-term survival depends on the ability to successfully induce a second remission and then consolidate with allo-HSCT. Allo-HSCT is the preferred therapy if HLA identical siblings or matched unrelated donors are available (Estey, 2000; Breems et al., 2005; Craddock et al., 2005; Döhner et al., 2010; Foran, 2010; O'Donnel et al., 2011). Although a prognostic index including remission duration, initial cytogenetics, prior HSCT or not, and age at relapse has been developed for younger patients with relapsed AML (Breems et al., 2005), the impact of molecular aberrations on allo-HSCT is still unclear in CN-AML patients beyond first remission. Since the prognostic impact of molecular aberrations on the outcomes of allo-HSCT remains uncertain, and studies from Asian population were still lacking, the aim of this study was to analyze CN-AML patients undergoing allo-HSCT to evaluate the impact of different molecular aberrations, including mutations of NPM1, FLT3-ITD, and CEBPA, on the clinical outcomes of allo-HSCT in the Taiwanese population.

\section{MATERIAL AND METHODS}

A total of 42 de novo non-M3 adult AML patients who received allo-HSCT from July 2002 to November 2009 were retrospectively reviewed in the Kaohsiung Medical University Hospital, a referral medical center in southern Taiwan. Therapy-related MDS/AML was excluded. The cytogenetic studies were performed on bone marrow samples using the conventional G-banding method when AML was first diagnosed. Among them, a normal karyotype was found in $23(54.7 \%)$ patients, forming the database for analysis after obtaining informed consent and approval from the Institutional Review Board of the Kaohsiung Medical University Hospital. All patients received standard I3A7 $\left(12 \mathrm{mg} / \mathrm{m}^{2}\right.$ idarubicin for 3 days and 100 $\mathrm{mg} / \mathrm{m}^{2}$ cytarabine for 7 days) induction chemotherapy after the diagnosis of AML. Failure to achieve remission after I3A7 was defined as induction failure. Six (26.1\%) patients were classified as induction failure and were given re-induction chemotherapy.

The disease status at the time of allo-HSCT was determined, and $11(47.8 \%)$ patients were in first remission, $5(21.7 \%)$ patients in second remission, and 7 (30.4\%) patients without remission. The median follow-up period was 104 weeks (range 8 to 384 weeks). All patients were engrafted successfully, and all patients received standard graft-versus-host disease (GVHD) prophylaxis with methotrexate and cyclosporine. The basic characteristics of the patients receiving allo-HSCT are shown in Table 1. 
Table 1. Basic characteristics of the CN-AML patients receiving allo-HSCT.

\begin{tabular}{lc}
\hline Characteristics & Number of patients $(\%, \mathrm{~N}=23)$ \\
\hline Age (years) & 41 \\
Median & $22-61$ \\
Range & $7(30.4)$ \\
Gender & $16(69.6)$ \\
Male & \\
Female & $6(26.1)$ \\
Failure of induction chemotherapy & $17(73.9)$ \\
Yes & $15(65.2)$ \\
No & $8(34.8)$ \\
Donor & $11(47.8)$ \\
HLA-identical sibling & $5(21.7)$ \\
Alternative & $7(30.4)$ \\
Disease status at the time of allo-HSCT & $21(91.3)$ \\
First remission & $2(8.7)$ \\
Second remission & $22(95.7)$ \\
No remission & $1(4.3)$ \\
Conditioning & $6(26.1)$ \\
Myeloablative & $17(73.9)$ \\
Reduced-intensity regimen & \\
TBI-containing regimen & 7.22 \\
Non-TBI regimen & $3.16-11.65$ \\
ATG-containing regimen & \\
Non-ATG-containing regimen & $14(60.9)$ \\
CD34 cells (x 10 $/ \mathrm{kg})$ & $9(39.1)$ \\
Median & Range \\
Acute GVHD & Grades 0-I \\
Grades II-IV & \\
\hline
\end{tabular}

$\mathrm{TBI}=$ total body irradiation; $\mathrm{ATG}=$ anti-thymocyte globulin; GVHD = graft-versus-host disease.

Genomic DNA isolated from mononuclear cells of bone marrow samples at the time of diagnosis of AML was analyzed to determine the mutational status of NPM1, CEBPA and FLT3-ITD in all CN-AML patients. Polymerase chain reaction (PCR) amplification of NPM1 exon 12 was carried out followed by direct sequencing for determination of NPM1 mutation status as previously described (Döhner et al., 2005). PCR was carried out and the products were resolved on a $2 \%$ agarose gel stained with ethidium bromide for determination of FLT3ITD mutation status (Fröhling et al., 2002). Four different sets of primers were used for PCR amplification followed by direct sequencing to determine the CEBPA-coding region mutation status, as previously described (Fröhling et al., 2004).

Categorical variables were analyzed using chi-square tests. Kaplan-Meier analysis was performed to estimate relapse-free survival and overall survival. The probabilities between subgroups were compared by the log-rank test. All statistics were calculated using the SPSS 11.5 software (SPSS Inc., Chicago, IL, USA). P $<0.05$ indicated a statistically significant difference.

\section{RESULTS}

Among the $23 \mathrm{CN}$-AML patients who received allo-HSCT, the NPM1 mutation was found in $7(30.4 \%)$ patients, FLT3-ITD in $3(13.0 \%)$ patients, and CEBPA mutation in $4(17.4 \%)$ patients. Among the 7 patients with the NPM1 mutation, 2 were found to have a concurrent FLT3-ITD mutation, and the other 5 patients did not have the FLT3-ITD mutation. 
Among the 4 patients with the CEBPA mutation, 1 had a double mutation, and none of them was found to have a concurrent FLT3-ITD mutation.

After induction chemotherapy with I3A7, 6 (26.1\%) patients failed to achieve remission. The NPM1 and CEBPA mutation status showed no association with remission rate (P $=0.99$ and 0.99 , respectively). More induction failure was found in patients with FLT3-ITD (66.7\%) compared with patients without FLT3-ITD (16.7\%), but no statistical significance was found $(\mathrm{P}=0.16)$.

The overall survival and relapse-free survival categorized by disease status at the time of allo-HSCT are shown in Figure 1. Patients in any remission status showed significantly better overall survival $(\mathrm{P}<0.001$, Figure 1A) and relapse-free survival $(\mathrm{P}=0.002$, Figure 1B) compared with patients who were not in remission at the time of allo-HSCT. Among patients in remission, the first remission and the second remission showed no clinical significance in terms of overall survival $(\mathrm{P}=0.31)$.

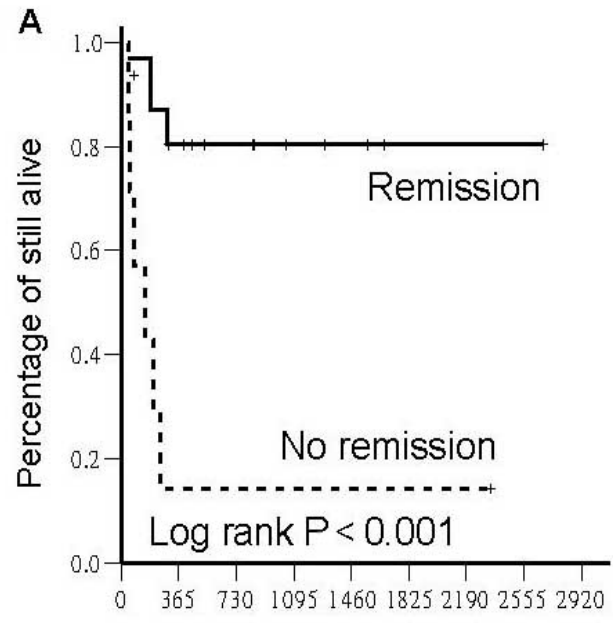

Days after HSCT

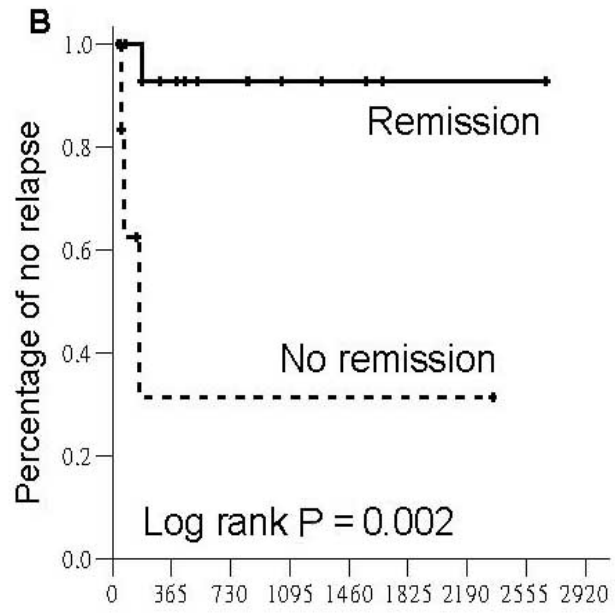

Days after HSCT

Figure 1. A. Overall survival and B. relapse-free survival of the CN-AML patients receiving allo-HSCT categorized by disease status at the time of allo-HSCT.

The overall survival after allo-HSCT categorized by different molecular aberrations is shown in Figure 2. When categorized into two risk groups by molecular aberrations, the patients in the good risk group (harboring either NPMI or CEBPA mutations without concurrent FLT3-ITD) showed a borderline significantly worse overall survival compared with the patients in the intermediate/poor risk group $(\mathrm{P}=0.08$, Figure $2 \mathrm{~A})$. No overall survival difference was found between patients with or without the $N P M 1$ mutation $(\mathrm{P}=0.96$, Figure 2B). Interestingly, a poorer overall survival was found in patients with the CEBPA mutation compared with patients without the $C E B P A$ mutation $(\mathrm{P}=0.003$, Figure $2 \mathrm{C})$. A poorer overall survival was found in patients with the FLT3-ITD mutation, but this did not reach statistical significance $(P=0.15$, Figure 2D). 
A
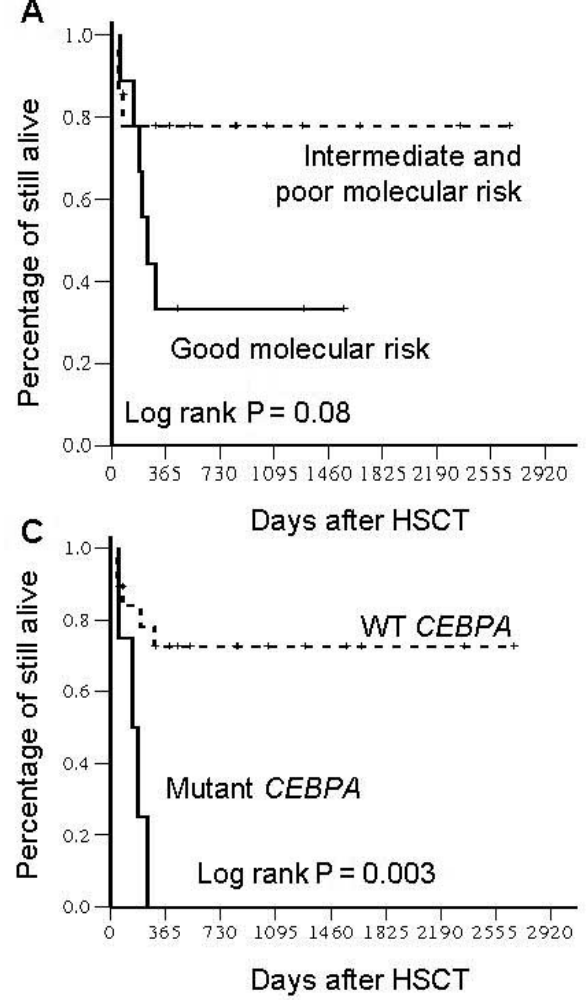

B

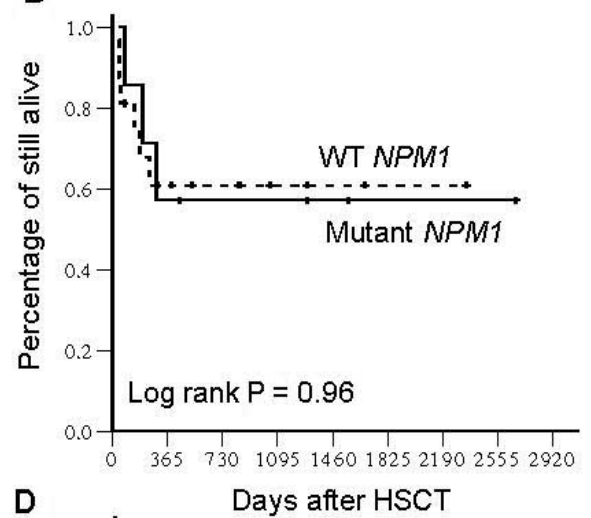

D

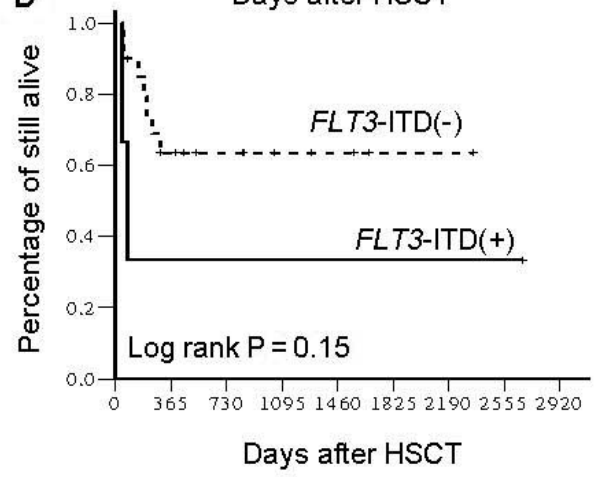

Figure 2. Kaplan-Meier survival estimates of the CN-AML patients receiving allo-HSCT categorized by genotype. A. Good molecular risk group versus intermediate/poor molecular risk group. B. NPM1 mutation versus NPM1 wild-type (WT). C. CEBPA mutation versus CEBPA WT. D. FLT3-ITD versus no FLT3-ITD.

Eleven patients received allo-HSCT at first remission, 4 of whom were in the good risk group and 7 in the intermediate/poor risk group. None of them was found to have FLT3ITD. Patients in the good risk group did not have any significant overall survival benefits after allo-HSCT, and an even worse overall survival compared with patients in the intermediate/ poor risk group $(\mathrm{P}=0.33$, Figure $3 \mathrm{~A})$. Two of the 4 patients in the good risk group and 1 of the 7 patients in intermediate/poor risk group died. The causes of death in the good risk group included 1 patient with acute GVHD and 1 patient who relapsed, with a treatment-related mortality (TRM) rate of $25 \%$. The cause of death in the intermediate/poor risk group was acute GVHD, with a TRM of $14.2 \%$.

In the 12 patients who received allo-HSCT beyond first remission, disease status played an important role on overall survival. Patients in second remission $(\mathrm{N}=5)$ had a significantly better overall survival compared with patients without remission $(\mathrm{N}=7, \mathrm{P}=$ 0.006 , Figure 3B). There was no statistical significance in overall survival between patients in the good risk group and the intermediate/poor risk group $(\mathrm{P}=0.17$, Figure $3 \mathrm{C})$. Similarly, no overall survival difference was found between patients with or without FLT3-ITD ( $\mathrm{P}=$ 0.28 , Figure 3D). 

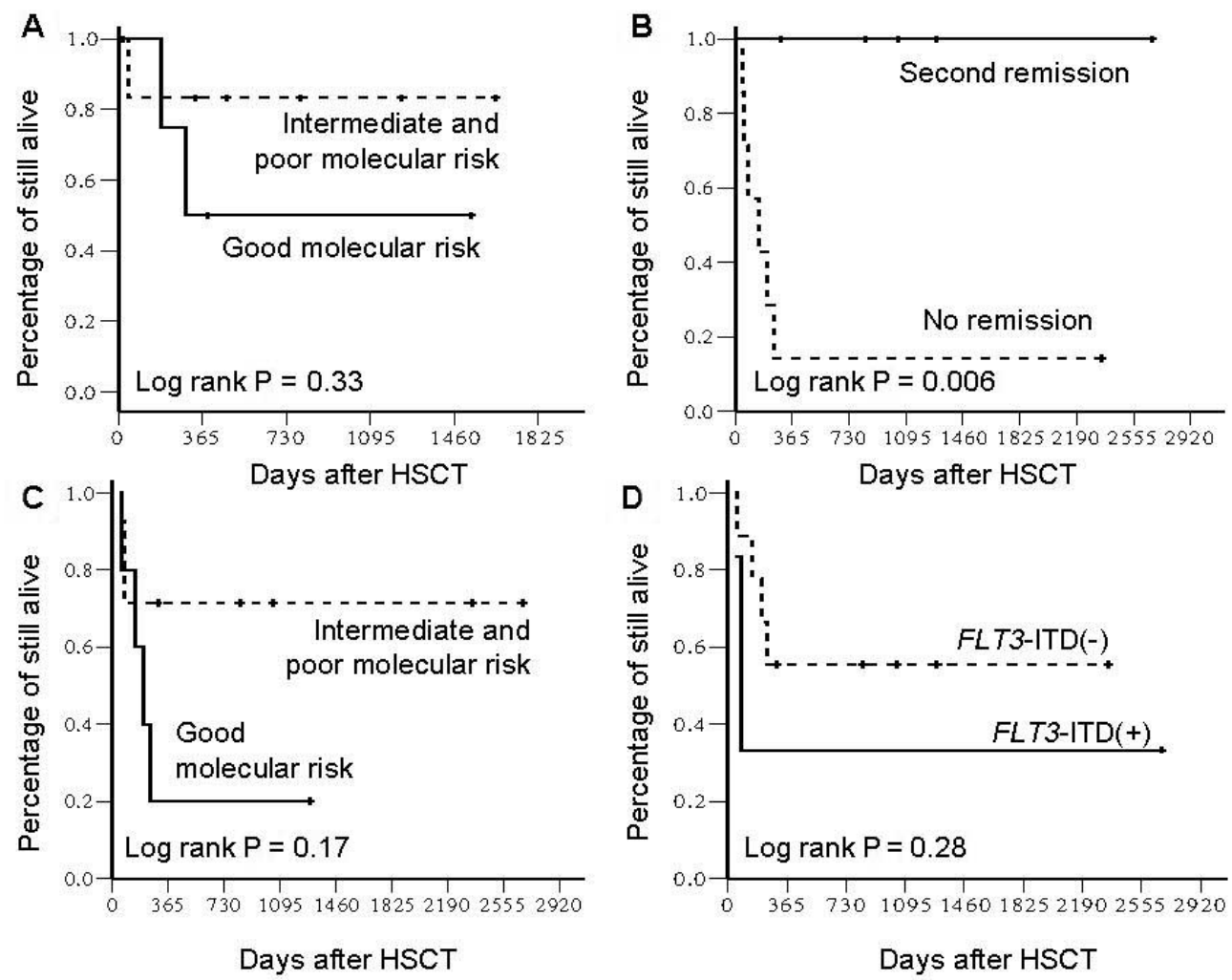

Figure 3. Kaplan-Meier survival estimates of the CN-AML patients receiving allo-HSCT at first remission (A) and beyond first remission (B-D) categorized by genotype. A. Patients in first remission, good molecular risk group versus intermediate/poor molecular risk group. B. Disease status at the time of allo-HSCT in patients beyond first remission. C. Patients beyond first remission, good molecular risk group versus intermediate/poor molecular risk group. D. Patients beyond first remission, FLT3-ITD versus no FLT3-ITD.

\section{DISCUSSION}

In this study focusing on the Taiwanese population, the good molecular risk group, including those with isolated NPMI or CEBPA mutations without FLT3-ITD, did not have an overall survival benefit after allo-HSCT compared to the intermediate/poor risk group, either in all patients or in patients in first remission. Interestingly, patients harboring isolated $C E B P A$ mutations had a significantly worse overall survival compared with those without $C E B P A$ mutations, but this was not seen in patients with isolated NPM1 mutations. No difference in overall survival was found in patients with or without FLT3-ITD. In patients receiving alloHSCT at first remission, those in the good risk group still had no overall survival benefits. In patients receiving allo-HSCT beyond the first remission, disease status played a major role irrespective of molecular aberrations.

Several large studies in Western populations have demonstrated that the NPM1 mutation without an additional FLT3-ITD mutation is associated with a better remission rate after 
induction chemotherapy and overall survival (Döhner et al., 2005; Schnittger et al., 2005; Thiede et al., 2006; Schlenk et al., 2008). To explore the role of allo-HSCT in patients with isolated NPM1 mutations, a "donor versus no-donor" comparison in post-remission therapy was performed by the German-Austrian AML study group, which found a similar outcome for patients independent of the availability of an HLA-matched sibling donor (Schlenk et al., 2008). In other genotypes (excluding mutant CEBPA), a survival benefit was found if a donor existed. This indicates that in patients with isolated NPM1 mutations, the role of allo-HSCT in first remission should be carefully evaluated.

The prognostic impact of $C E B P A$ mutations has also been evaluated by several study groups from Western populations. Similar to NPM1 mutations, isolated CEBPA mutations without additional FLT3-ITD was associated with better clinical outcomes including remission duration and overall survival in several studies (Preudhomme et al., 2002; Fröhling et al., 2004), but the impact on allo-HSCT has seldom been analyzed. Our study revealed a poorer outcome in patients with the CEBPA mutation receiving allo-HSCT, even though none of them had FLT3-ITD. Some factors may be considered for such condition. First, only one patient with an isolated $C E B P A$ mutation $(25 \%)$ had a double mutation, and the others had a single mutation. Several studies have shown that $C E B P A$-double but not $C E B P A$-single mutation patients have a better overall survival (Green et al., 2010; Taskesen et al., 2011). Second, more patients with the CEBPA mutation were in non-remission status at the time of allo-HSCT. Although this interpretation should be cautious due to the limited case number, the role of alloHSCT should be carefully evaluated in patients with isolated CEBPA mutations.

Most studies have demonstrated an adverse prognostic factor in patients with the FLT3-ITD mutation, but whether allo-HSCT is beneficial for patients with the FLT3-ITD mutation is controversial. In one study, no survival benefit was found in patients with FLT3-ITD undergoing allo-HSCT based on a "donor versus no-donor" comparison (Gale et al., 2005). In contrast, a reduced risk of relapse was found for patients with FLT3-ITD undergoing alloHSCT in another study (Bornhäuser et al., 2007). In our analysis, patients with FLT3-ITD had a relatively worse overall survival, but this did not reach statistical significance. However, in patients beyond first remission, there was no survival difference between patients with or without FLT3-ITD, indicating that allo-HSCT may be able to overcome the adverse impact of FLT3-ITD. Since most studies have been retrospective, prospective studies are necessary to clarify the prognostic effect of allo-HSCT in patients with the FLT3-ITD mutation.

The frequency of NPM1, FLT3-ITD, and CEBPA mutations in our cohort was similar to studies in Western populations, suggesting that an ethnic difference is not obvious. There were no significant associations between NPMI and CEBPA mutations and failure of induction chemotherapy in our analysis, compatible with previous studies (Preudhomme et al., 2002; Fröhling et al., 2004; Döhner et al., 2005; Schnittger et al., 2005; Thiede et al., 2006; Schlenk et al., 2008). In patients with FLT3-ITD, the remission rate after induction chemotherapy is controversial (Kottaridis et al., 2001; Schnittger et al., 2002; Fröhling et al., 2002; Thiede et al., 2002). In our patients with FLT3-ITD, including mutant NPM1 with FLT3-ITD, we observed a relatively poorer but not statistically significant remission rate after induction chemotherapy, although these cases were limited in our cohort.

Disease status at the time of allo-HSCT remained an important prognostic factor for overall survival in our study. In patients achieving first remission after induction chemotherapy, those with an intermediate/poor risk had a better survival than patients with a good risk, 
indicating the importance of allo-HSCT as post-remission therapy in intermediate/poor risk patients. In patients beyond first remission, the impact of molecular aberrations on allo-HSCT is unclear. In one study, FLT3-ITD and age were independently negative prognostic factors for survival in relapsed CN-AML patients, including patients who underwent allo-HSCT after relapse (Wagner et al., 2011). The NPMI and CEBPA mutation status had no impact on survival after relapse, but the impact on allo-HSCT was not reported. In our study, disease status was the main determinant for overall survival, indicating the importance of entering second remission before allo-HSCT. Interestingly, survival was better in patients with an intermediate/poor risk than in patients with a good risk, indicating that allo-HSCT may be able to overcome the relatively poor outcome in intermediate/poor risk patients beyond first remission.

In summary, no survival benefits were found from allo-HSCT in our CN-AML patients with a good molecular risk, including patients in first remission. Furthermore, patients with $C E B P A$ mutations showed a significantly worse overall survival, suggesting that the role of allo-HSCT in good molecular risk patients should be carefully evaluated, especially in patients with CEBPA mutations. Conversely, allo-HSCT should be considered in first remission in patients with an intermediate/poor risk, and it may overcome the adverse impact of FLT3ITD. In patients beyond first remission, disease status was the main issue for overall survival after allo-HSCT, irrespective of molecular aberrations.

\section{REFERENCES}

Bornhäuser M, Illmer T, Schaich M, Soucek S, et al. (2007). Improved outcome after stem-cell transplantation in FLT3/ ITD-positive AML. Blood 109: 2264-2265.

Breems DA, Van Putten WL, Huijgens PC, Ossenkoppele GJ, et al. (2005). Prognostic index for adult patients with acute myeloid leukemia in first relapse. J. Clin. Oncol. 23: 1969-1978.

Byrd JC, Mrozek K, Dodge RK, Carroll AJ, et al. (2002). Pretreatment cytogenetic abnormalities are predictive of induction success, cumulative incidence of relapse, and overall survival in adult patients with de novo acute myeloid leukemia: results from Cancer and Leukemia Group B (CALGB 8461). Blood 100: 4325-4336.

Craddock C, Tauro S, Moss P and Grimwade D (2005). Biology and management of relapsed acute myeloid leukaemia. Br. J. Haematol. 129: 18-34.

Döhner K, Schlenk RF, Habdank M, Scholl C, et al. (2005). Mutant nucleophosmin (NPM1) predicts favorable prognosis in younger adults with acute myeloid leukemia and normal cytogenetics: interaction with other gene mutations. Blood 106: 3740-3746.

Döhner H, Estey EH, Amadori S, Appelbaum FR, et al. (2010). Diagnosis and management of acute myeloid leukemia in adults: recommendations from an international expert panel, on behalf of the European LeukemiaNet. Blood 115: 453-474.

Estey EH (2000). Treatment of relapsed and refractory acute myelogenous leukemia. Leukemia 14: 476-479.

Foran JM (2010). New prognostic markers in acute myeloid leukemia: perspective from the clinic. Hematology. Am. Soc. Hematol. Educ. Program 2010: 47-55.

Fröhling S, Schlenk RF, Breitruck J, Benner A, et al. (2002). Prognostic significance of activating FLT3 mutations in younger adults (16 to 60 years) with acute myeloid leukemia and normal cytogenetics: a study of the AML Study Group Ulm. Blood 100: 4372-4380.

Fröhling S, Schlenk RF, Stolze I, Bihlmayr J, et al. (2004). CEBPA mutations in younger adults with acute myeloid leukemia and normal cytogenetics: prognostic relevance and analysis of cooperating mutations. J. Clin. Oncol. 22: 624-633.

Gale RE, Hills R, Kottaridis PD, Srirangan S, et al. (2005). No evidence that FLT3 status should be considered as an indicator for transplantation in acute myeloid leukemia (AML): an analysis of 1135 patients, excluding acute promyelocytic leukemia, from the UK MRC AML10 and 12 trials. Blood 106: 3658-3665.

Green CL, Koo KK, Hills RK, Burnett AK, et al. (2010). Prognostic significance of CEBPA mutations in a large cohort of younger adult patients with acute myeloid leukemia: impact of double CEBPA mutations and the interaction with FLT3 and NPM1 mutations. J. Clin. Oncol. 28: 2739-2747. 
Grimwade D, Walker H, Oliver F, Wheatley K, et al. (1998). The importance of diagnostic cytogenetics on outcome in AML: analysis of 1,612 patients entered into the MRC AML 10 trial. The Medical Research Council Adult and Children's Leukaemia Working Parties. Blood 92: 2322-2333.

Koreth J, Schlenk R, Kopecky KJ, Honda S, et al. (2009). Allogeneic stem cell transplantation for acute myeloid leukemia in first complete remission: systematic review and meta-analysis of prospective clinical trials. JAMA 301: 2349-2361.

Kottaridis PD, Gale RE, Frew ME, Harrison G, et al. (2001). The presence of a FLT3 internal tandem duplication in patients with acute myeloid leukemia (AML) adds important prognostic information to cytogenetic risk group and response to the first cycle of chemotherapy: analysis of 854 patients from the United Kingdom Medical Research Council AML 10 and 12 trials. Blood 98: 1752-1759.

Mrozek K, Heerema NA and Bloomfield CD (2004). Cytogenetics in acute leukemia. Blood Rev. 18: 115-136.

O’Donnell MR, Abboud CN, Altman J, Appelbaum FR, et al. (2011). Acute myeloid leukemia. J. Natl. Compr. Canc. Netw. 9: 280-317.

Preudhomme C, Sagot C, Boissel N, Cayuela JM, et al. (2002). Favorable prognostic significance of CEBPA mutations in patients with de novo acute myeloid leukemia: a study from the Acute Leukemia French Association (ALFA). Blood 100: 2717-2723.

Schlenk RF, Döhner K, Krauter J, Frohling S, et al. (2008). Mutations and treatment outcome in cytogenetically normal acute myeloid leukemia. N. Engl. J. Med. 358: 1909-1918.

Schnittger S, Schoch C, Dugas M, Kern W, et al. (2002). Analysis of FLT3 length mutations in 1003 patients with acute myeloid leukemia: correlation to cytogenetics, FAB subtype, and prognosis in the AMLCG study and usefulness as a marker for the detection of minimal residual disease. Blood 100: 59-66.

Schnittger S, Schoch C, Kern W, Mecucci C, et al. (2005). Nucleophosmin gene mutations are predictors of favorable prognosis in acute myelogenous leukemia with a normal karyotype. Blood 106: 3733-3739.

Taskesen E, Bullinger L, Corbacioglu A, Sanders MA, et al. (2011). Prognostic impact, concurrent genetic mutations, and gene expression features of AML with CEBPA mutations in a cohort of 1182 cytogenetically normal AML patients: further evidence for CEBPA double mutant AML as a distinctive disease entity. Blood 117: 2469-2475.

Thiede C, Steudel C, Mohr B, Schaich M, et al. (2002). Analysis of FLT3-activating mutations in 979 patients with acute myelogenous leukemia: association with FAB subtypes and identification of subgroups with poor prognosis. Blood 99: 4326-4335.

Thiede C, Koch S, Creutzig E, Steudel C, et al. (2006). Prevalence and prognostic impact of NPM1 mutations in 1485 adult patients with acute myeloid leukemia (AML). Blood 107: 4011-4020.

Wagner K, Damm F, Thol F, Gohring G, et al. (2011). FLT3-internal tandem duplication and age are the major prognostic factors in patients with relapsed acute myeloid leukemia with normal karyotype. Haematologica 96: 681-686. 